\title{
On statistical equilibrium in helical fluid flows
}

\author{
M. V. Kurgansky ${ }^{1, *}$ \\ ${ }^{1}$ Department of Geophysics, Faculty of Physical and Mathematical Sciences, University of Concepción, Casilla 160-C, \\ Concepción, Chile \\ *on leave from: A. M. Obukhov Institute of Atmospheric Physics, Russian Academy of Sciences, Moscow, Russia
}

Received: 20 December 2005 - Revised: 30 March 2006 - Accepted: 3 April 2006 - Published: 2 June 2006

\begin{abstract}
The statistical mechanics of 3-D helical flows is re-examined for a continuum truncated at a top wavenumber. Based on the principle of equipartition of the flow enstrophy between helical modes, the emerging (i) energy spectrum law " -2 " and (ii) formal mathematical analogy between the helicity and the thermodynamic entropy are discussed. It is noted that the " -2 " scaling law is consistent with both spectral equilibrium and spectral cascade paradigms. In an attempt to apply the obtained results to a turbulent flow regime within the Earth's outer liquid core, where the net helicity of a turbulent flow component is presumably explained by Earth's rotation, it has been noticed that it is the energy spectral law " -1 ", but not " -2 ", which is likely realized there and within the logarithmic accuracy corresponds to the case of the velocity structure function $[u(l)]^{2}$ independency on the spatial scale $l$, the latter is consistent with observations. It is argued that the " -1 " scaling law can also be interpreted in terms of the spectral equilibrium and it is emphasized that the causes of the likely dominance of the spectral law " -1 " over the spectral law " -2 " in this geophysical application deserve further investigation and clarification.
\end{abstract}

\section{Introduction}

By writing this article I would like to commemorate the scientific work by S. S. Moiseev, who was one of the pioneers in systematic applications of the helicity concept to geophysical fluid flows. His bold idea on possibility of the "kinetic alphaeffect" and the "kinetic dynamos", had been put forward in a series of publications beginning with Moiseev et al. (1983a, b), has become a long-lasting inspiration to the author of these lines. However, I would like to dedicate this article to a somewhat different topic but also related to the helicity con-

Correspondence to: M. V. Kurgansky

(kurgansk@udec.cl) cept and had been in the focus of S. S. Moiseev's scientific interests as well (cf. Moiseev and Chkhetiani, 1996), namely to an issue of the energy and the helicity spectral distribution in fluid flows possessing the net helicity and being viewed as an ensemble of interacting helical Beltrami flows, or helical modes hereafter. In the forthcoming, a particular emphasis will be placed on the flow enstrophy and its distribution between helical modes of different spatial scales and also on that presumably important role, which the enstrophy spectral distribution may play in specifying the entire statistical regime in helical flows.

\section{Preliminaries}

Consider an inviscid 3-D incompressible fluid flow, which is spatially homogeneous and isotropic but lacks of mirror symmetry. This flow is described by equations

$\partial_{t} \boldsymbol{v}+\nabla\left(\boldsymbol{v}^{2} / 2\right)+(\nabla \times \boldsymbol{v}) \times \boldsymbol{v}=-\nabla(p / \rho), \quad \nabla \cdot \boldsymbol{v}=0,(1)$

where $v$ is the velocity vector, $p$ is the pressure and $\rho$ is the uniform density. In Cartesian coordinates with the position vector $\boldsymbol{x}$, consider a flow within a periodic box $V(D)$ of size $D$. In this set-up Eq. (1) possess an infinite countable set of steady Beltrami solutions (cf. Levich, 1987; Dritschel, 1991) satisfying the equations

$\nabla \times \boldsymbol{u}_{k}^{ \pm}= \pm k \boldsymbol{u}_{k}^{ \pm}, \quad \nabla \cdot \boldsymbol{u}_{k}^{ \pm}=0, \quad k>0$.

Helical modes (2) are normalized by unity, $\left\langle\left(\boldsymbol{u}_{k}^{ \pm}\right)^{2}\right\rangle \equiv$ $D^{-3} \iiint_{V(D)}\left(\boldsymbol{u}_{k}^{ \pm}\right)^{2} d \boldsymbol{x}=1$, and always allow taking the general solution of (1) in the form (Knorr et al., 1990; Waleffe, 1992) $\boldsymbol{v}(\boldsymbol{x}, t)=\sum_{k}\left[a_{k}(t) \boldsymbol{u}_{k}^{+}(\boldsymbol{x})+b_{k}(t) \boldsymbol{u}_{k}^{-}(\boldsymbol{x})\right]$, where $a_{k}(t), b_{k}(t)$ are time-dependent amplitudes. The kinetic energy, the helicity and the enstrophy of the fluid flow $\operatorname{read} E=\left\langle\boldsymbol{v}^{2}\right\rangle / 2=\sum_{k}\left(a_{k}^{2}+b_{k}^{2}\right) / 2, H=\langle\boldsymbol{v} \cdot \nabla \times \boldsymbol{v}\rangle / 2=$

Published by Copernicus GmbH on behalf of the European Geosciences Union and the American Geophysical Union. 
$\sum_{k} k\left(a_{k}^{2}-b_{k}^{2}\right) / 2$ and $F=\left\langle(\nabla \times v)^{2}\right\rangle / 2=\sum_{k} k^{2}\left(a_{k}^{2}+b_{k}^{2}\right) / 2$, respectively. These formulas can symbolically be rewritten as $E=\sum_{k}\left(E_{k}^{+}+E_{k}^{-}\right), H=\sum_{k}\left(H_{k}^{+}+H_{k}^{-}\right)$, and $F=\sum_{k}\left(F_{k}^{+}+F_{k}^{-}\right)$, where $F_{k}^{+}=k H_{k}^{+}=k^{2} E_{k}^{+}$and $F_{k}^{-}=-$ $k H_{k}^{-}=k^{2} E_{k}^{-}$. We further introduce the relative weight $0 \leq q_{k} \leq 1$ of helical modes with the positive helicity by writing $E_{k}^{+}=q_{k} E_{k}$ and $E_{k}^{-}=\left(1-q_{k}\right) E_{k}$; therefore $E=\sum_{k} E_{k}$, $H=\sum_{k} H_{k} \equiv \sum_{k}\left(2 q_{k}-1\right) k E_{k}$, and $F=\sum_{k} F_{k} \equiv \sum_{k} k^{2} E_{k}$.

\section{Spectral equilibrium and "helicity-entropy" analogy}

The integrals $E, H$ and $F$ satisfy the Schwarz inequality $H^{2} \leq E F$ (Moffatt, 1969), which sets a lower bound on the enstrophy value $F \geq F_{\min }=H^{2} / E$. On the other hand, one may assume that in fluid flows with small but finite viscosity the scale-selective Newtonian viscosity virtually does not violate the energy and the helicity conservation law, especially because the sign-indefiniteness of the helicity dissipation function, but leads to maximum possible reduction of the flow enstrophy $F$ values, consistent with Moffatt's lower bound. The kinetic energy viscous dissipation rate $\varepsilon$, being proportional to $F$, tends to minimum in this case. This $\varepsilon$-property may be thought as a manifestation of the minimum entropy production principle in non-equilibrium thermodynamics and also as a special assumption concerning $\varepsilon$ behavior in turbulent flows with the non-zero helicity. Therefore, though being not precisely a constant of motion, the enstropy $F$ in helical flows may have close each other upper and lower bounds on its possible time-variations. This pseudoconservation property of the flow ensrophy $F$ is laid into the basis of proceeding arguments appealing to the ideas of equilibrium statistical fluid dynamics (Salmon, 1998; Chapter 5) and finally leading to the notion of equipartition of the flow enstrophy between helical modes. One has to be much more cautious in applying these ideas to our case than in the classical cases of 3-D reflexion-invariant turbulence or 2-D turbulence, where the energy equipartition principle or the principle of equipartition of a linear combination of energy and enstrophy is used, respectively. On the other hand, the principle of equipartition of the flow enstrophy between helical modes, per se, i.e. when it is considered as a basing principle, could be of a certain heuristic value. A similar situation occurs, e.g., with regard to the principle of equipartition between the kinetic energy and the magnetic energy in magnetohydrodynamics (cf. Elsasser, 1955), which enables one to obtain simple but useful estimates in many interesting cases. The point, however, is how to find conditions of such basing principles validity and how to define an area of applicability of them. Only the critical comparison, and resulting conformity, of those deductions which can be inferred based upon the proposed principle with the experimental or numerical modeling results may lead to progress in that direction.

After this preamble, we proceed with formal arguments, applied to our problem. A matter of fact, for Beltrami flows (2) the enstrophy becomes a constant of motion, which value is fixed by the equality $F=H^{2} / E$ given the inviscid constants of motion $E$ and $H$; therefore, the enstrophy related invariant probabilistic measure can be introduced. Consider an infinite ensemble of flow realizations (replicas), every replica being a steady Beltrami flow described by (2) and having one and the same $F$-value. Now, the probability $p_{k}=F_{k} / F$ (see Sect. 2 for notations) for a randomly chosen replica to have a given spatial scale, $k^{-1}$, fully specifies the helicity and the energy spectral distribution via the formulas $H_{k}=F k^{-1}\left(2 q_{k}-1\right) p_{k}$ and $E_{k}=F k^{-2} p_{k}$. Different Beltrami flow patterns of either helicity sign but having the same $k$-value are indistinguishable within this approach and are ascribed equal a priori probabilities. We truncate our fluid system at the minimum $k_{0}$ and maximum $k_{\max }$ wave numbers, where $k_{0}$ is high enough that the modes are dense (mode spacing small compared with $k$ ). Afterwards, we replace discrete probabilities $p_{k}$ and factors $q_{k}$ with the probability density function $p(k)$ and continuous weight function $q(k)$, such that $\int_{k_{0}}^{k_{\max }} p(k) d k=1$, $H=\int_{k_{0}}^{k_{\max }} \hat{H}(k) d k \equiv F \int_{k_{0}}^{k_{\max }}[2 q(k)-1] p(k) k^{-1} d k$, and $E=\int_{k_{0}}^{k_{\max }} \hat{E}(k) d k \equiv F \int_{k_{0}}^{k_{\max }} p(k) k^{-2} d k$. Following the ideas of equilibrium statistical fluid dynamics (see also Burgers, 1939), it is anticipated that the total enstrophy is equipartitioned between modes, having spatial scales falling into the indicated $k$-range. This also corresponds to a general method of statistical inference by Jaynes (1957) based on the Shannon informational entropy maximization, in our case: $\quad \int_{k_{0}}^{k_{\max }}-\log p(k) p(k) d k=\max$ provided $\int_{k_{0}}^{k_{\max }} p(k) d k=1$. It implies $p(k)=\left(k_{\max }-k_{0}\right)^{-1}=\mathrm{constant}$ and $\chi \equiv F p(k)=F\left(k_{\max }-k_{0}\right)^{-1}=$ constant, where $\chi$ has the dimension of helicity density. These have two consequences. First, energy spectral distribution reads

$\hat{E}(k)=\chi k^{-2} \equiv \chi k^{\frac{-6}{3}}$

and occupies an intermediate position between the classical Kolmogorov-Obukhov " $-5 / 3$ " and helicity cascade “-7/3" (Brissaud et al., 1973) scaling laws. Kraichnan (1959) pointed out and discussed the spectral law (3) but from a different viewpoint, in no way related to helicity. The spectral law " -2 " also corresponds to an idealized fluid flow, considered as an ensemble of tangential discontinuities ("fronts") in the velocity field (cf. Scorer, 1997); besides, this scaling law emerges in rapidly rotating fluids (Zhou, 1995; see also below). Second, under an assumption $\delta(k) \equiv 2 q(k)-1=$ $A /\left(k_{1}+k\right)$, with $A$ and $k_{1}$ as constants, one obtains the 
formulas

$$
\begin{aligned}
& H=A \chi \int_{k_{0}}^{k_{\max }}\left(k_{1}+k\right)^{-1} k^{-1} d k=\frac{A \chi}{k_{1}} \log \left[\frac{k_{\max }\left(k_{1}+k_{0}\right)}{k_{0}\left(k_{1}+k_{\max }\right)}\right] \\
& E=\chi \int_{k_{0}}^{k_{\max }} k^{-2} d k=\chi\left(k_{0}^{-1}-k_{\max }^{-1}\right) .
\end{aligned}
$$

Two limit cases are of interest. First, $k_{0}<<k_{\max }<<k_{1}$, when $\delta(k)$ is quasi-constant throughout the interval $\left[k_{0}, k_{\max }\right]$ and formulas (4) yield $H \cong\left(A \chi / k_{1}\right) \log (L / \lambda)$, $E=\chi(L-\lambda) \cong \chi L$, where the notations $L=k_{0}^{-1}$ and $\lambda=k_{\max }^{-1}$ are introduced. The above expressions resemble classical formulas for the entropy $S=c \log T+$ constant and the internal energy $U=c T$, taken for isochoric processes; here, $T$ is the absolute (Kelvin) temperature and $c$ is the heat capacity. In the light of this analogy, it might be conjectured that the helicity $H$ is an entropy-like variable, whereas the maximum cut-off scale $L$ divided by the helical asymmetry factor, $\delta_{0} \equiv \delta(0)=A / k_{1} \leq 1$, is the temperature analogue. This analogy is lost when $\delta_{0}=0$, i.e. the flow is nonhelical. Also, the most fundamental temperature definition reads $T^{-1}=d S / d U$ (Landau and Lifshitz, 1999), whereas in the taken limit case it follows from (4) that $\left(L / \delta_{0}\right)^{-1}=$ ( $\partial H / \partial E)\left.\right|_{\lambda=\text { constant }}$. Second limit case $k_{0}<<k_{1}<<k_{\max }$ corresponds to a realistic situation when the net helicity is maximal at the largest scales but a flow is nearly nonhelical at the smallest scales (cf. Ditlevsen and Giuliani, 2001). Now, it follows from (4) that $H \cong\left(A \chi / k_{1}\right) \log \left(k_{1} L\right)$, $E=\chi(L-\lambda) \cong \chi L$, which only re-scales the helicity but maintains the thermodynamic analogy. In a particular case of $k_{1}^{2}=k_{0} k_{\max }$ one simply gets $H \cong\left(A \chi / 2 k_{1}\right) \log (L / \lambda)$. Finally, for $k_{1}<<k \leq k_{\max }$ our model predicts the same " -2 " scaling law for both the helicity and the energy spectral distribution, in a certain analogy with the notion of helicity cascade linearity (cf. Ditlevsen and Giuliani, 2001).

\section{Discussions and some geophysical implications}

The results of Section 3 find some support in the direct numerical simulation (DNS) of the decay of turbulence by Holm and Kerr (2002). Their diagnostics showed that the initial value problem for turbulence evolves through an early stage, with still negligible dissipation, when vortex sheets are formed from smooth initial conditions and afterwards they roll up into helical ("strongly Beltramized") vortex tubes. In this context, it is worth mentioning again that the spectral law " -2 " exactly corresponds to a fluid flow considered as an ensemble of vortex sheets. Once helical vortex tubes have formed, viscous dissipation grows rapidly in the DNS and the energy spectrum gradually approaches the classical $k^{-5 / 3}$ characteristic of a turbulent energy cascade, but before it is accomplished, the energy spectrum remains steeper than "-5/3"; see Fig. 6 in Holm and Kerr (2002). Once a clear "-5/3" spectrum appears, the energy decay becomes self-similar. In the light of these DNS results, it may be conjectured that the spectral law (3) is most relevant to a nearly inviscid fluid flow, whose dynamics is dominated by both energy and helicity conservation. The latter flow most closely corresponds to early stages of the decay of turbulence starting from helical initial conditions. Later on, the viscous dissipation becomes significant and the downscale energy cascade develops, where the helicity plays a secondary role compared to the energy (cf. Kraichnan, 1973).

In a real Navier-Stokes fluid, the minimum cut-off scale $\lambda$, which enters $H$-definition in Sect. 3 and provides $H$ the properties of a coarse-grained quantity, can on pure dimensional grounds be expressed through the fluid viscosity $v$ and the basic parameter $\chi$ by the formula $\lambda \sim \sqrt[3]{v^{2} / \chi}$. On the other hand, it can be inferred that $\chi \cong F \lambda$ and $\varepsilon=2 \nu F \cong$ $2 \nu \chi / \lambda$, for increasingly small but still finite $\nu$-values. Elimination of $\lambda$ between these two estimates yields $\chi \sim \sqrt[4]{\varepsilon^{3} / v}$, namely a formula which is "reciprocally dual" to the Kolmogorov microscale $\eta \sim \sqrt[4]{v^{3} / \varepsilon}$ definition.

We see that arguments of Sect. 3 allow for a formal analogy between the helicity and the thermodynamic entropy. Kraichnan (1973) considered the thermodynamics of absolute equilibrium of the helical turbulence in an inviscid 3-D truncated set-up, but from a different viewpoint, based on the Gibbs statistics, energy \& helicity conservation principles and without any use of the enstrophy $F$. Despite (3) is quite different from the absolute equilibrium spectra by Kraichnan (1973) our results are in accordance with his main conclusion that strong net helicity depresses overall turbulent energy transfer. Also, Kraichnan's (1973) words are worth to be cited, namely that the absolute equilibrium ensembles of course are very far from the actual states of viscous turbulence and that their value is in pointing to direction in which the actual, non-equilibrium state may be plausibly expected to transfer excitation.

From an alternative, direct energy cascade perspective, the spectrum (3) is consistent with the concept of an inertial range, which requires $1<n<3$ for spectra obeying the $k^{-n}$. law (Kraichnan, 1959). It is slightly steeper than the Kolmogorov spectrum and it would be interesting to search for hints on (3) in observational or numeric simulation data (see also above).

In general cases, for a turbulent helical flow characterized by the constant energy downscale cascade rate $\varepsilon$, the corresponding helicity downscale cascade rate $\sigma(l)$ taken for some spatial scale $l$ can, on pure dimensional grounds and by the use of the "local-cascade-approximation", be expressed in the form $\sigma(l) \sim[h(l)]^{3} / \varepsilon$. Here, $h(l)$ is the helicity per unit volume dominated at the same spatial scale $l$. This parameterization of course is not unique, but it attracts with its simplicity and, what is probably most important, the obtained cubic dependency of $\sigma(l)$ on $h(l)$ is perfect 


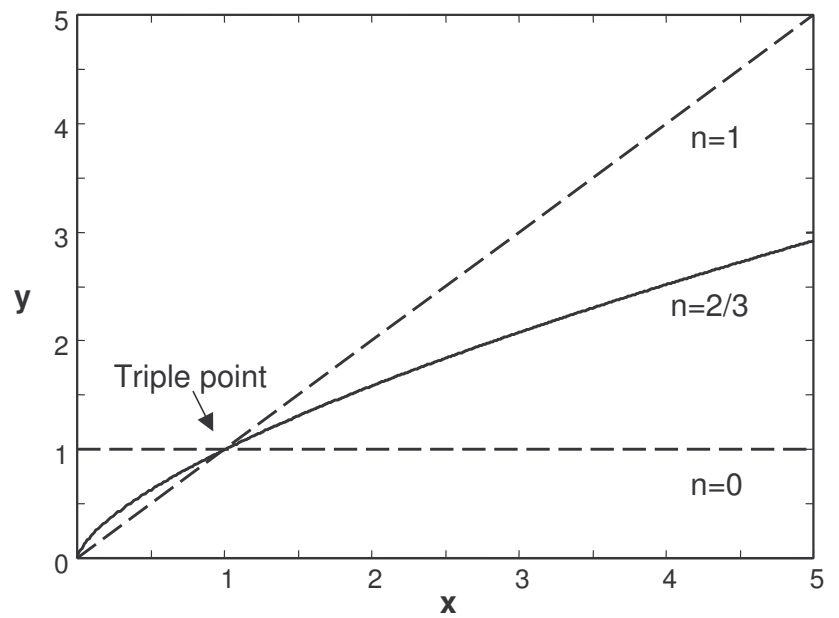

Fig. 1. Schematic of the intersection between the graphs of three different structure functions $[u(l)]^{2}=C_{n} u_{*}^{2}\left(l / l_{*}\right)^{n}$, for $n=0,2 / 3,1$ (a horizontal dashed, a solid and an inclined dashed line, respectively), occurring in a single triple point for $C_{n}=C=1$. The abscissa and the ordinate of any point in the graph are measured in $x=l / l_{*}$ and $y=\left[u(l) / u_{*}\right]^{2}$, respectively; the definitions of both $l_{*}$ and $u_{*}$ are given in the text.

from the general symmetry viewpoint, merely because both $\sigma(l)$ and $h(l)$ are pseudo-scalar quantities. Now, a request of the helicity flux constancy, $\sigma(l)=$ constant, implies that $h(l) \sim$ constant. Using an estimate $|h(l)| \sim[u(l)]^{2} / l$, where $u(l)$ is the characteristic turbulent velocity at the scale $l$, one gets $[u(l)]^{2} \propto l$, consistent with the spectrum $\hat{E}(k) \propto k^{-2}$, see (3). We conclude that both spectral equilibrium and spectral cascade paradigms give practically identical results in the considered case of turbulent fluid flows with the net helicity. In performing the above estimates we use an approximation of the helicity only as a relation between the velocity and the spatial scale, thus following the existing tradition in helical turbulence studies (cf. Moffatt, 1978), though this approximation has its inherent limitations and should be applied to helical turbulence with major precautions.

In rotating fluids, the overall fast fluid rotation may provide a turbulent flow the net helicity and also makes the flow spatially anisotropic. In this analysis, we account for the former property but neglect the latter one, for the sake of simplicity. In this case and when based on the existing experience of studying the helicity balance for the Ekman boundary layer flow (see Kurgansky, 2002 and references therein), one can assume that $\sigma(l) \sim \Omega h(l)$, where $\Omega$ is the angular velocity of fluid rotation; in other words, $\Omega^{-1}$ stands for the characteristic time of helicity destruction in weakly viscous and rapidly rotating fluids. Comparison between $\sigma(l) \sim[h(l)]^{3} / \varepsilon$ and $\sigma(l) \sim \Omega h(l)$ leads to the formula $[h(l)]^{2} \sim \varepsilon \Omega$, which together with the estimate $|h(l)| \sim[u(l)]^{2} / l$ yields $[u(l)]^{2} \sim l \sqrt{\varepsilon \Omega}$. The last expression is consistent with the " -2 " spectral scaling law by Zhou
(1995) and finds its direct experimental support in Baroud et al. (2002).

One of the most prominent areas of these results application would be the study of helical turbulent flows within the Earth's outer liquid core, where $\varepsilon \sim 10^{-12} \mathrm{~m}^{2} \mathrm{~s}^{-3}$ (Jacobs, 1987-1991; Golitsyn, 1991), $\Omega \sim 10^{-4} \mathrm{~s}^{-1}$ and, hence, $\sqrt{\varepsilon \Omega} \sim 10^{-8} \mathrm{~ms}^{-2}$. However, direct application of the above derived formulas yields $u(l) \sim 10^{-1} \mathrm{~ms}^{-1}$ at the largest scale $l \sim 10^{6} \mathrm{~m}$, which by three orders of magnitude exceeds the usually assumed value $u(l) \sim 10^{-4} \mathrm{~ms}^{-1}$, had been derived from the terrestrial magnetic field secular variations analysis based upon the frozen-in magnetic field hypothesis (cf. Bloxham and Jackson, 1991). We conclude that only very small spatial scales of motion, if at all, are relevant to the $[u(l)]^{2} \sim l \sqrt{\varepsilon \Omega}$ scaling law. Also, an application of the Kolmogorov " $2 / 3$ " scaling law, $[u(l)]^{2} \sim(\varepsilon l)^{2 / 3}$, gives $u(l) \sim 10^{-2} \mathrm{~ms}^{-1}$ for the above indicated value $l \sim 10^{6} \mathrm{~m}$. On the other hand, for large spatial scales, where the Rossby number becomes increasingly small, the direct energy cascade rate might be specified by a quantity $\varepsilon \sim[u(l)]^{2} / \tau(l)$, with $\tau(l) \sim \Omega^{-1}$ replacing the turbulent eddy turnover time $\tau(l) \sim l / u(l)$ in the Kolmogorov theory. In this way, we obtain $[u(l)]^{2} \sim \varepsilon / \Omega \sim 10^{-8} \mathrm{~m}^{2} \mathrm{~s}^{-2}$, which gives a correct order-of-magnitude estimate of the observed fluid velocities within the Earth's outer core (cf. Golitsyn, 1991). Kurgansky (1995) used this estimate and also an independence of the turbulent velocity on the spatial scale as a plausible scenario to construct a dissipative dynamo model. If to use the notations $l_{*} \equiv \sqrt{\varepsilon / \Omega^{3}}, u_{*} \equiv \sqrt{\varepsilon / \Omega}$, then the above written three different formulas for $[u(l)]^{2}$ can be presented in a universal form $[u(l)]^{2}=C_{n} u_{*}^{2}\left(l / l_{*}\right)^{n}$, where $n=0,2 / 3,1$ and $C_{n}=O$ (1) are non-dimensional constants. When $C_{n}=C=1$, then the corresponding graphs intersect in a single point $O$ having coordinates $l=l_{*},[u(l)]^{2}=u_{*}^{2}$; this $O$-point can conventionally be named the triple point (see, Fig. 1). If the constants $C_{n}$ were different but still sufficiently close each other, then the triple point $O$ would split into three separate points positioned in the vertices of a small triangle $A B C$ centered at $O$. For the Earth's outer core the characteristic spatial scale, $l_{*} \sim 10^{0} \mathrm{~m}$, is very small. The Rossby number $R o(l) \equiv u(l) / \Omega l \sim\left(l / l_{*}\right)^{-1+n / 2}$ plays the role of a control parameter in the problem. For $l<l_{*}, R o(l)>1$ and rotation is not dynamically important; therefore, the Kolmogorov regime with $n=2 / 3$ emerges. At $l=l_{*}$, when also $R o \sim 1$, the "bifurcation" occurs and the fluid flow must choose between two alternative regimes, either with $n=1$ or with $n=0$. As it is evident from inspecting of Fig. 1, these two regimes cannot co-exist and, most likely, the regime with $n=0$ dominates within the Earth's outer core, when the fall of power spectrum of the kinetic energy is significantly slower relative to the Kolmogorov spectrum (cf. Reshetnyak, 2005). This likely lack of "available room" for the " -2 " energy spectrum (corresponding to $n=1$ and $[u(l)]^{2} \propto l$ ) in the turbulent motion within the Earth's liquid core is surprising and 
needs future explanation. We only note that an assumption for its existence at $l<l_{*}$ would be physically controversial because, in any case, the Kolmogorov regime must emerge for the finest spatial scales.

Because the turbulent flow regime with $n=0$ plays, seemingly, such an important role in the Earth's outer core, it is instructive to re-visit arguments of Section 3 and to investigate how this case can be explained in terms of the spectral equilibrium. To do it, we specify the Beltrami flows via the Helmholtz vector equation

$\nabla^{2} \boldsymbol{u}_{k}^{ \pm}+k^{2} \boldsymbol{u}_{k}^{ \pm}=0, \quad \nabla \cdot \boldsymbol{u}_{k}^{ \pm}=0$

that follows from (2). In their turn, Eqs. (2) factorize (5). Prior to work of Knorr et al. (1990) and Waleffe (1992), Eq. (5) was the standard way to specify the Beltrami flows. Contrary to (2), Eq. (5) does not distinguish between Beltrami flows of different helicity sign. Keeping this in mind, consider a discrete probability distribution for Beltrami flows over $k^{2}$-values (but not $k$-values as in Sect. 3 !), provided all flow replicas possess the same enstrophy $F$-value. After that, for a sufficiently dense set of discrete $k^{2}$-values has been truncated at the wavenumbers $k_{0}^{2}$ and $k_{\max }^{2}$, we introduce a continuous probability density function $\tilde{p}\left(k^{2}\right)$, such that $\int_{k_{0}^{2}}^{k_{\max }^{2}} \tilde{p}\left(k^{2}\right) d k^{2}=1$. By applying the maximum entropy principle we deduce that $\tilde{p}\left(k^{2}\right)=\left(k_{\max }^{2}-k_{0}^{2}\right)^{-1}=$ constant; also, $\tilde{\chi} \equiv F\left(k_{\max }^{2}-k_{0}^{2}\right)^{-1}=$ constant has now the physical dimension of velocity squared. In an analogy to the arguments of Sect. 3 one gets $\hat{E}(k)=2 F \tilde{p}\left(k^{2}\right) k^{-1} \equiv 2 \tilde{\chi} k^{-1}$ and the total kinetic energy of a flow reads

$E=\int_{k_{0}}^{k_{\max }} \hat{E}(k) d k=2 \tilde{\chi} \log \left(k_{\max } / k_{0}\right) \equiv 2 \tilde{\chi} \log (L / \lambda)$,

which within the logarithmic accuracy is independent on the scale $L$, consistent with the previous arguments.

One may be tempted to conjecture that the case of $n=1$, with basic helical modes given by (2), allows for an analogy with a fermionic quantum system, whereas the case of $n=0$, corresponding to (5), is analogous to a bosonic case of two helical modes having the same $k$-value but opposite helicity signs and constituting a couple with zero net helicity, the latter in a definite analogy to the coupling of two fermionic particles occurring in superconductors. The classical Kolmogorov turbulence, corresponding to $n=2 / 3$, is merely non-helical. In the light of this analogy, it can also be added that Eq. (2) manifest themselves as a distant analogue of Dirac's quantum mechanical equations, whereas Eq. (5) have some resemblance to the relativistic Schrödinger equation.

\section{Conclusions}

In this article, the equilibrium statistical mechanics of 3-D helical flows is re-examined for a continuum truncated at a top wavenumber. Based on the principle of equipartition of the flow enstrophy between helical modes, the emerging energy spectrum law "-2" and a formal mathematical analogy between the helicity, on the one hand, and the thermodynamic entropy, on the other hand, are discussed. It is noted that the " -2 " scaling law is consistent with both spectral equilibrium and spectral cascade paradigms.

An attempt was made to apply the obtained results to a turbulent flow regime within the Earth's outer core, where the net helicity of a turbulent flow component is presumably explained by Earth's rotation. Somewhat surprisingly, it was noticed that it is the energy spectral law " -1 ", but not "-2", which is likely realized for core motions. This $k^{-1} \mathrm{spec}$ tral law within the logarithmic accuracy corresponds to the velocity structure function $[u(l)]^{2}$, which is independent on the spatial scale $l$ (cf. (6)), the latter is consistent with observations. It was argued that the " -1 " scaling law can also be interpreted in terms of the spectral equilibrium and was emphasized that the causes of the likely dominance of the spectral law "-1" over the spectral law " -2 " in this geophysical application deserve future investigation and clarification.

Acknowledgements. I am grateful to O. G. Chkhetiani for useful discussions and to anonymous referees for constructive comments which led to several improvements of the manuscript.

Edited by: N. S. Erokhine

Reviewed by: five referees

\section{References}

Baroud, C. N., Plapp, B. B., She, Z.-S. and Swinney, H. L.: Anomalous self-similarity in a turbulent rapidly rotating fluid, Phys. Rev. Lett., 88, 11, 114501, doi:10.1103/PhysRevLett.88.114501, 2002.

Bloxham, J. and Jackson, A.: Fluid flow near the surface of Earth's outer core, Rev. Geophys., 29, 1, 97-120, 1991.

Brissaud, A., Frisch, U., Leorat, J., Lesieur, M., and Mazure, A.: Helicity cascades in fully developed isotropic turbulence, Phys. Fluids, 16, 1366-1377, 1973.

Burgers, J. M.: Mathematical examples illustrating relations occurring in the theory of turbulent fluid motion, Hon. Ned. Acad. Wet. Vern. Eerste Sectie., 1939, D1, XVII, 2, 1-53, 1939.

Ditlevsen, P. D. and Giuliani, P.: Dissipation in helical turbulence, Phys. Fluids, 13, 11, 3508-3509, 2001.

Dritschel, D. G.: Generalized helical Beltrami flows in hydrodynamics and magnetohydrodynamics, J. Fluid. Mech., 222, 525$541,1991$.

Elsasser, W. M.: Hydromagnetism. 1. A review, Am. J. Phys., 23, 590-609, 1955.

Golitsyn, G. S.: Convection regimes on various geophysical and astrophysical objects, Izvestiya, Atmos. Ocean. Phys., 27, 20 31, 1991.

Holm, D. D. and Kerr, R.: Transient vortex events in the initial value problem for turbulence, Phys. Rev. Lett., 88, 24, 244501, doi:10.1103/PhysRevLett.88.244501, 2002. 
Jacobs, J. A.: Geomagnetism (4 volumes), Academic Press, New York, 1987-1991.

Jaynes, E. T.: Information theory and statistical mechanics, Phys. Rev., 106, 4, 620-630, 1957.

Knorr, G., Lynov, J. P., and Pécseli, H. P: Self-organization in three-dimensional hydrodynamic turbulence, Z. Naturforsch, 45a, 1059-1073, 1990.

Kraichnan, R. H.: The structure of isotropic turbulence at very high Reynolds numbers, J. Fluid Mech., 5, 497-543, 1959.

Kraichnan, R. H.: Helical turbulence and absolute equilibrium, J. Fluid Mech., 59, 745-752, 1973.

Kurgansky, M. V.: On the problem of magnetic field generation by helical turbulence, Geophys. Astrophys. Fluid Dyn., 81, 101112, 1995.

Kurgansky, M. V.: Adiabatic invariants in large-scale atmospheric dynamics, Taylor \& Francis, London and New York, 2002.

Moiseev, S. S. and Chkhetiani, O. G.: Helical scaling in turbulence, Journal of Experimental and Theoretical Physics, 83, 1, 192198, 1996.

Moiseev, S. S., Sagdeev, R. Z., Tur, A. V., Khomenko, G. A., and Shukurov, A. M.: Physical mechanism of amplification of vortex disturbances in the atmosphere, Sov. Phys. Dokl., 28, 925-928, 1983a.

Moiseev, S. S., Sagdeev, R. Z., Tur, A. V., Khomenko, G. A., and Yanovsky, V. V.: A theory of large-scale structure origination in hydrodynamic turbulence, Sov. Phys. JETF, 58, 1149-1157, $1983 b$.
Moffatt, H. K.: The degree of knottendness of tangled vortex lines, J. Fluid. Mech., 35, 1, 117-129, 1969.

Moffatt, H. K.: Magnetic field generation in electrically conducting fluids, Cambridge Univ. Press, Cambridge, 1978.

Landau, L. D. and Lifshitz, E. M.: Statistical physics, Vol. 5, Landau and Lifshitz course of theoretical physics, ButterworthHeinemann, Oxford, UK, 1999.

Levich, E.: Certain problems in the theory of developed hydrodynamical turbulence, Physics Reports (Review Section of Physics Letters), 151, 129-238, 1987.

Reshetnyak, M. Yu.: Estimation of turbulent viscosity of the Earth's liquid core, Doklady Earth Sciences, 400, 1, 84-88, 2005.

Salmon, R.: Lectures on geophysical fluid dynamics, Oxford University Press, New York, Oxford, 1998.

Scorer, R. S.: Dynamics of meteorology and climate, John Wiley \& Sons in association with Praxis Publishing, Chichester, England, 1997.

Waleffe, F.: The nature of triad interactions in homogeneous turbulence, Phys. Fluids A, 4, 350-363, 1992.

Zhou, Y.: A phenomenological treatment of rotating turbulence, Phys. Fluids, 7, 8, 2092-2094, 1995. 